\title{
ULTRAFAST INFRARED AND VISIBLE SPECTROSCOPY OF INTERMOLECULAR ELECTRON TRANSFER FROM DIMETHYL ANILINE TO COUMARIN 337
}

\author{
BORIS AKHREMITCHEV, CHENGFEI WANG \\ and GILBERT C. WALKER* \\ Department of Chemistry, University of Pittsburgh, \\ Pittsburgh, PA 15260, USA
}

(Received 7 April 1997)

\begin{abstract}
Ultrafast visible and infrared polarization spectroscopy has been used to reveal the orientation of donor and acceptor molecules in the intermolecular electron transfer between coumarin 337 and dimethylaniline.
\end{abstract}

Keywords: Electron transfer; ultrafast dynamics; vibrational relaxation; solvent effects; intermolecular electron transfer

Recent efforts have focused on the role of non-equilibrium configurations of the solvent and the solute in electron transfer [1-2]. Intermolecular charge transfer reactions between a solute and a solvent may provide a direct probe of the first solvent-shell because of the rapid decay with distance of the electron tunneling probability. We have performed ultrafast pump experiments on the intermolecular electron transfer between coumarin 337 (C337) solute and dimethylaniline (DMA) solvent. The electron transfer process is illustrated in Figure 1.

We have performed $400 \mathrm{~nm}$ pump, $500 \mathrm{~nm}$ probe measurements, see Figure 2. The anisotropies taken at early $(0.2 \mathrm{ps})$ and late (10 and $30 \mathrm{ps}$ ) times indicate that the $\mathrm{C} 337$ gain spectrum results from the same

\footnotetext{
* Corresponding author.
} 


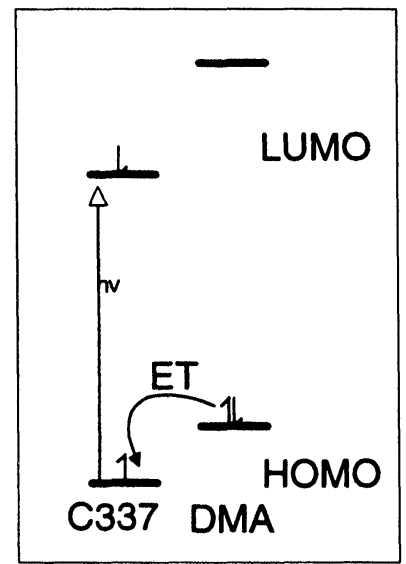

FIGURE 1 Following photoexcitation of C337, electron transfer from DMA to C337 occurs.

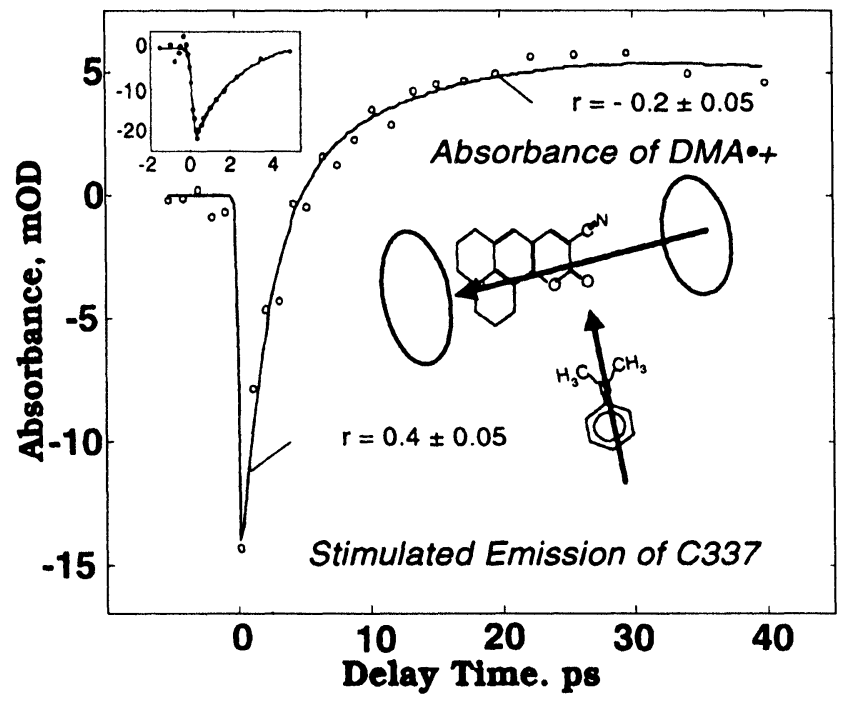

FIGURE 2 Transient absorption of C337 in DMA. $400 \mathrm{~nm}$ pump $/ 500 \mathrm{~nm}$ probe.

transition as the optically pumped transition $(r=0.4 \pm 0.05)$, and a new, DMA radical cation absorption exhibits a transition moment nearly perpendicular to the pumped transition, $r=-0.2 \pm 0.05$. 


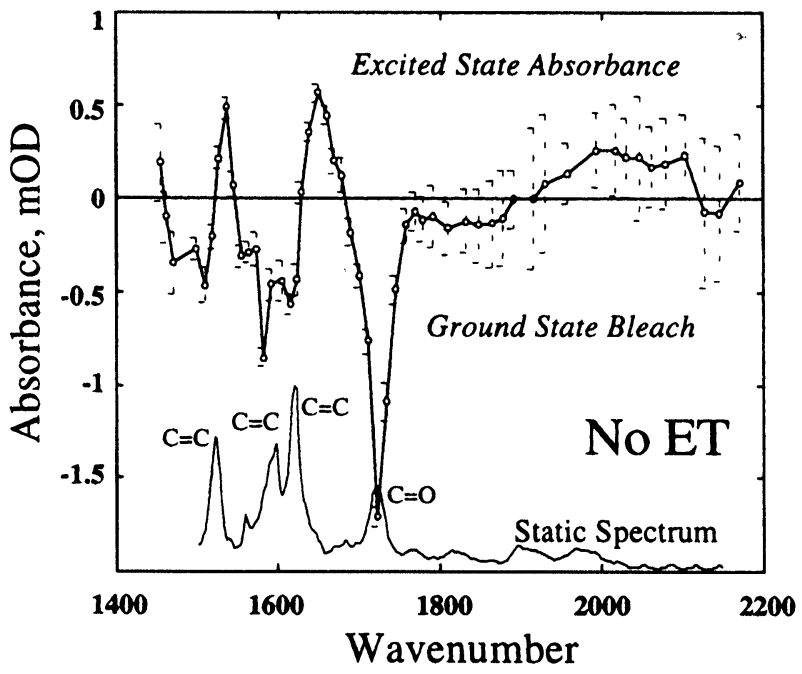

FIGURE 3 C337 in Benzonitrile. Static and 5 ps delay after $400 \mathrm{~nm}$ pump spectra.

We have also performed visible pump IR probe experiments to reveal the $\mathrm{S}_{0}-\mathrm{S}_{1}$ transition moment versus the carbonyl and nitrile stretch absorption transition moments. (see Fig. 3.) . These data indicate that the electronic transition moment direction of $\mathrm{C} 337$ is long axis polarized. The transition moment direction of the dimethylaniline radical cation absorption lies parallel to the DMA long axis. Therefore, the anisotropy indicates that the electron donor and acceptor molecules are aligned with their long axes roughly perpendicular. C337 and DMA have $10 \mathrm{D}$ and $1.6 \mathrm{D}$ ground state dipole moments, respectively, aligned roughly along their long axes. The observed anisotropy indicates that electron transfer is rate controlled by favorable overlap of the HOMO's of C337 and DMA, which is not necessarily optimized by the attractive and repulsive intermolecular interactions.

\section{References}

[1] For recent reviews in this area see the centennial issue of J. Phys. Chem., 1996 Vol. 100.

[2] Nagasawa, Y. Yartsev, A. P. Tominaga, K. Bisht, P. B. Johnson, A. E. and Yoshihara, K. (1995). J. Phys. Chem., 99, 653. 